\title{
Erratum to: Genetic variability and phenotypic plasticity of apple morphological responses to soil water restriction in relation with leaf functions and stem xylem conductivity
}

Pierre-Éric Lauri ${ }^{1,7}$ • Têtè Sévérien Barigah ${ }^{2,6}$ - Gerardo Lopez ${ }^{1,3}$.

Sébastien Martinez ${ }^{1}$ - Pasquale Losciale ${ }^{4} \cdot$ Marco Zibordi $^{3} \cdot$ Luigi Manfrini $^{3}$.

Luca Corelli-Grappadelli ${ }^{3} \cdot$ Evelyne Costes $^{1} \cdot$ Jean-Luc Regnard $^{5}$

Published online: 9 August 2016

(C) Springer-Verlag Berlin Heidelberg 2016

\section{Erratum to: Trees}

\section{DOI 10.1007/s00468-016-1408-3}

Unfortunately, some part text of Table 3 was missing in the original publication of this article. The complete corrected Table 3 is given in the following page.

The online version of the original article can be found under doi:10.1007/s00468-016-1408-3.

\section{Pierre-Éric Lauri}

lauri@supagro.inra.fr

1 INRA, UMR AGAP, 34398 Montpellier, Cedex 05, France

2 INRA, UMR 547 PIAF, 63100 Clermont-Ferrand, France

3 Dipartimento Scienze Agrarie, University of Bologna, V.le G, Fanin 46, 40127 Bologna, Italy

4 Consiglio per la ricerca in agricoltura e l'analisi dell'economia agraria (CREA), Research Unit for Agriculture in Dry Environments [CREA-SCA], Bari, Italy

5 Montpellier SupAgro, UMR AGAP, 34060 Montpellier, Cedex 02, France

6 Clermont Université, Université Blaise-Pascal, UMR 547 PIAF, BP 10448, 63000 Clermont-Ferrand, France

7 Present Address: UMR System, Campus Montpellier SupAgro, 2 place Pierre Viala, Bât. 27, 34060 Montpellier, France 
Table 3 Whole-shoot response to drought of two apple cultivars 'Starkrimson' and 'Granny Smith' and in 19 of their progenies, during two consecutive periods of growth (I and II)

\begin{tabular}{|c|c|c|c|c|c|c|c|}
\hline \multirow{3}{*}{$\begin{array}{l}\text { Period and water } \\
\text { regime }\end{array}$} & \multicolumn{6}{|c|}{ Whole-shoot-response to drought } & \multirow{3}{*}{$\begin{array}{l}\text { Effects of factors } \\
\left(F \text { or } \chi^{2}, P\right)\end{array}$} \\
\hline & \multicolumn{2}{|l|}{ Low } & \multicolumn{2}{|l|}{ Medium } & \multicolumn{2}{|l|}{ High } & \\
\hline & Values & MD/WWI (\%) & Values & MD/WWI (\%) & Values & MD/WWI (\%) & \\
\hline \multicolumn{8}{|l|}{ Period I } \\
\hline No. of genotypes & \multicolumn{2}{|l|}{7} & \multicolumn{2}{|l|}{7} & \multicolumn{2}{|l|}{7} & \\
\hline \multicolumn{8}{|l|}{ WWI } \\
\hline TLA & $646.4 \pm 201.5$ & - & $709.3 \pm 194.0$ & - & $635.3 \pm 109.2$ & - & $0.37,0.69$ \\
\hline $\mathrm{L}$ & $26.7 \pm 6.5$ & - & $32.8 \pm 3.8$ & - & $27.5 \pm 7.3$ & - & $2.13,0.15$ \\
\hline PercGC & $28.5 \pm 18.5$ & - & $7.6 \pm 13.5$ & - & $21.7 \pm 0.19$ & - & $3.3,0.05$ \\
\hline$g_{\mathrm{s}}$ & $337.2 \pm 40.0$ & - & $370.8 \pm 41.5$ & - & $360.5 \pm 52.8$ & - & $1.02,0.38$ \\
\hline SWP & $-0.8 \pm 0.1$ & - & $-0.8 \pm 0.0$ & - & $-0.8 \pm 0.1$ & - & $0.44,0.65$ \\
\hline$P_{\mathrm{n}}$ & $11.3 \pm 1.0$ & - & $11.4 \pm 0.9$ & - & $11.2 \pm 1.2$ & - & $0.07,0.93$ \\
\hline PLC & - & - & - & - & - & - & - \\
\hline \multicolumn{8}{|l|}{$\mathrm{MD}$} \\
\hline TLA & $323.9 \pm 89.4 a$ & 50.1 & $221.6 \pm 60.7 b$ & 31.2 & $121.9 \pm 37.1 \mathrm{c}$ & 19.2 & $16.42,8.7310^{-5}$ \\
\hline $\mathrm{L}$ & $17.0 \pm 4.6 \mathrm{a}$ & 63.2 & $13.4 \pm 1.6 b$ & 40.9 & $7.7 \pm 3.6 \mathrm{c}$ & 28.0 & $12.37,4.1810^{-4}$ \\
\hline PercGC & $41.5 \pm 33.6 b$ & 145.6 & $50.2 \pm 23.2 \mathrm{ab}$ & 660.5 & $80.9 \pm 17.8 \mathrm{a}$ & 372.8 & $15.76,3.8010^{-4}$ \\
\hline$g_{\mathrm{s}}$ & $289.2 \pm 35.7$ & 85.8 & $284.3 \pm 27.7$ & 76.7 & $275.6 \pm 37.7$ & 76.5 & $0.29,0.75$ \\
\hline SWP & $-1.2 \pm 0.2$ & 145.4 & $-1.1 \pm 0.3$ & 143.7 & $-1.1 \pm 0.2$ & 145.2 & $0.33,0.72$ \\
\hline$P_{\mathrm{n}}$ & $10.1 \pm 0.8$ & 89.4 & $9.6 \pm 0.6$ & 84.2 & $9.8 \pm 1.3$ & 87.5 & $0.19,0.83$ \\
\hline PLC & - & - & - & - & - & - & - \\
\hline \multirow{3}{*}{$\begin{array}{l}\text { Period and water } \\
\text { regime }\end{array}$} & \multicolumn{6}{|c|}{ Whole-shoot-response to drought } & \multirow{3}{*}{$\begin{array}{l}\text { Effects of factors } \\
\left(F \text { or } \chi^{2}, P\right)\end{array}$} \\
\hline & \multicolumn{2}{|l|}{ Low } & \multicolumn{2}{|l|}{ Medium } & \multicolumn{2}{|l|}{ High } & \\
\hline & Values & SD/WWII $(\%)$ & Values & SD/WWII $(\%)$ & Values & SD/WWII $(\%)$ & \\
\hline \multicolumn{8}{|l|}{ Period II } \\
\hline No. of genotypes & 7 & & 7 & & 7 & & \\
\hline \multicolumn{8}{|l|}{ WWII } \\
\hline TLA & $1252.7 \pm 163.1$ & - & $1424.6 \pm 301.7$ & - & $1347.7 \pm 340.6$ & - & $0.99,0.39$ \\
\hline $\mathrm{L}$ & $42.3 \pm 6.8$ & - & $45.4 \pm 7.8$ & - & $42.6 \pm 4.5$ & - & $0.48,0.63$ \\
\hline PercGC & $9.5+18.9$ & - & $7.1 \pm 13.1$ & - & $7.6 \pm 9.6$ & - & $3.17,0.20$ \\
\hline$g_{\mathrm{s}}$ & $360.6 \pm 63.3$ & - & $387.9 \pm 38.1$ & - & $358.3 \pm 27.4$ & - & $0.91,0.42$ \\
\hline SWP & $-0.9 \pm 0.1$ & - & $-0.9 \pm 0.1$ & - & $-0.9 \pm 0.1$ & - & $1.45,0.26$ \\
\hline$P_{\mathrm{n}}$ & - & - & - & - & - & - & - \\
\hline PLC & $3.2 \pm 3.1 \mathrm{a}$ & - & $4.7 \pm 6.9 \mathrm{a}$ & - & $2.2 \pm 1.4 \mathrm{~b}$ & - & $71.86,2.4910^{-16}$ \\
\hline \multicolumn{8}{|l|}{$\mathrm{SD}$} \\
\hline TLA & $448.5 \pm 88.1 \mathrm{a}$ & 35.8 & $309.7 \pm 76.1 b$ & 20.2 & $163.7 \pm 74.0 \mathrm{c}$ & 12.1 & $22.38,1.3110^{-5}$ \\
\hline $\mathrm{L}$ & $20.8 \pm 4.7 \mathrm{a}$ & 49.3 & $16.2 \pm 3.0 \mathrm{a}$ & 35.6 & $8.4 \pm 3.0 \mathrm{~b}$ & 19.7 & $21.02,1.9610^{-5}$ \\
\hline PercGC & $41.5 \pm 17.2$ & 435.7 & $38.1 \pm 28.4$ & 533.3 & $67.7 \pm 22.8$ & 889.1 & $5.12,0.08$ \\
\hline$g_{\mathrm{s}}$ & $212.2 \pm 55.1$ & 58.9 & $193.9 \pm 30.8$ & 50.0 & $163.5 \pm 23.6$ & 45.6 & $2.80,0.09$ \\
\hline SWP & $-1.5 \pm 0.1$ & 176.4 & $-1.7 \pm 0.3$ & 189.0 & $-1.6 \pm 0.2$ & 168.8 & $1.44,0.26$ \\
\hline$P_{\mathrm{n}}$ & - & - & - & - & - & - & - \\
\hline PLC & $15.2 \pm 9.8 \mathrm{a}$ & 475.4 & $15.8 \pm 6.3 \mathrm{a}$ & 332.1 & $11.8 \pm 10.1 b$ & 539.0 & $53.71,2.1710^{-12}$ \\
\hline
\end{tabular}

For each class of response (low, medium, high) absolute values (mean \pm SD) of shoot morphology and plant functioning traits are provided. For each trait, values with different letters indicate significant differences $(P<0.05)$ between means (ANOVA with the class of response as factor, followed when significant by HSD-Tukey multiple mean comparison test). The ratios MD/WWI in period I and SD/WWII in period II are also provided for each trait. Units of the variables are figured in Table 1 